\title{
5-azacytidine promotes terminal differentiation of hepatic progenitor cells
}

\author{
YUN HE ${ }^{1-4}$, JIEJIE CUI ${ }^{2,3}$, TONGCHUAN HE ${ }^{2-4}$ and YANG BI ${ }^{1-4}$ \\ ${ }^{1}$ Department of Pediatric Surgery; ${ }^{2}$ Stem Cell Biology and Therapy Laboratory; \\ ${ }^{3}$ Ministry of Education Key Laboratory of Child Development and Disorders, \\ ${ }^{4}$ Chongqing Stem Cell Therapy Engineering Technical Center, The Children's Hospital \\ of Chongqing Medical University, Chongqing 400014, P.R. China
}

Received August 20, 2014; Accepted March 24, 2015

DOI: $10.3892 / \mathrm{mmr} .2015 .3772$

\begin{abstract}
C) is known to induce cardiomyocyte differentiation. However, its function in hepatocyte differentiation is unclear. The present study investigated the in vitro capability of 5 -azaC to promote maturation and differentiation of mouse embryonic hepatic progenitor cells, with the aim of developing an approach for improving hepatic differentiation. Mouse embryonic hepatic progenitor cells (HP14.5 cells) were treated with 5-azaC at concentrations from 0 to $20 \mu \mathrm{mol} / 1$, in addition to hepatocyte induction culture medium. Hepatocyte induction medium induces HP14.5 cell differentiation. 5-azaC may enhance the albumin promotor-driven Gaussia luciferase (ALB-GLuc) activity in induced HP14.5 cells. In the present study $2 \mu \mathrm{mol} / 1$ was found to be the optimum concentration with which to achieve this. The expression of hepatocyte-associated factors was not significantly different between the group treated with 5-azaC alone and the control group. The mRNA levels of ALB; cytokeratin 18 (CK18); tyrosine aminotransferase (TAT); and cytochrome p450, family 1 , member A1 (CYP1A1); in addition to the protein levels of ALB, CK18 and uridine diphosphate glucuronyltransferase 1A (UGT1A) in the induced group with 5-azaC, were higher than those in the induced group without 5-azaC, although no significant differences were detected in expression of the hepatic stem cell markers, DLK and $\alpha$-fetoprotein, between the two groups. Treatment with 5-azaC alone did not affect glycogen synthesis or indocyanine green (ICG) metabolic function in HP14.5 cells, although it significantly increased ICG uptake and periodic acid-Schiff-positive cell numbers amongst HP14.5 cells. Therefore, the present study demonstrated that treatment with 5 -azaC alone exerted no effects on the
\end{abstract}

Correspondence to: Professor Yang Bi, Department of Pediatric Surgery, The Children's Hospital of Chongqing Medical University, 136 Zhongshan Er Road, Chongqing 400014, P.R. China

E-mail: yangbi1981@cqmu.edu.cn

Key words: 5-azacytidine, differentiation, hepatic progenitor cells maturation and differentiation of HP14.5 cells. However, 5 -azaC exhibited a synergistic effect on the terminal differentiation of induced hepatic progenitor cells in association with a hepatic induction medium.

\section{Introduction}

Liver transplantation, a surgical procedure used to replace a diseased liver with a healthy liver allograft, is the most commonly used technique for the treatment of liver failure and end-stage liver disease $(1,2)$. Due to the limitations of this form of treatment, including a shortage of donor organs, high technical difficulty and the requirement for lifelong immunosuppression, cell therapy-based treatment strategies have been developed $(3,4)$. Terminally differentiated hepatocytes exhibit powerful liver function of detoxification, metabolism and synthesis. However, their availability and low expansion efficiency in vitro are significant obstacles to hepatocyte transplantation (5). Hepatic progenitor cells (HPCs) are bipotential stem cells, which arise in the liver and are capable of differentiation into either hepatocytes or cholangiocytes, under the appropriate conditions. Embryonic HPCs exhibit self-renewal and differentiation potential, in addition to low immunogenicity, indicating that they may be a useful alternative source of hepatocytes $(6,7)$. Although a number of researchers have reported that hepatic progenitor cells are able to differentiate in vitro and in vivo into hepatic cells with certain function, the differentiation efficiency of these cells for use as a transplantation substitute remains unclear $(8,9)$. Therefore, it is necessary to develop techniques to stably and efficiently obtain mature functional hepatocytes from hepatic progenitor cells.

5-azacytidine (5-azaC) is one of multiple DNA methylase inhibitors that is able to reverse the methylation status of a gene, and restore its expression (10), and is currently the only known effective chemical compound with which to induce the differentiation of mesenchymal stem cells (MSCs) into myocardial cells $(11,12)$. Changes in DNA methylation status affect the differentiation of stem cells $(13,14)$. The derivation of hepatic progenitor cells from embryonic fetal liver cells is of value in the study of early human liver organogenesis, as well as in the creation of an unlimited source of donor cells for hepatocyte transplantation therapy (15). In the present study, it was 
Table I. RT-PCR primers (5'-3').

\begin{tabular}{lll}
\hline Gene & \multicolumn{1}{c}{ Forward } & \multicolumn{1}{c}{ Reverse } \\
\hline GAPDH & GGCTGCCCAGAACATCAT & CGGACACATTGGGGGTAG \\
DLK & GCTGGGACGGGAAATTCT & AACCCAGGTGTGCAGGAG \\
AFP & ACGAGGAAAGCCCCTCAG & GCCATTCCCTCACCACAG \\
ALB & CCAGACATTCCCCAATGC & CAAGTTCCGCCCTGTCAT \\
CK18 & CTGGGCTCTGTGCGAACT & ACAGAGCCACCCCAGACA \\
TAT & ACCTTCAATCCCATCCGA & TCCCGACTGGATAGGTAG \\
CYP7A1 & GATTCTGATGCTGTCTTACTT & CAATATCATTTAGTGGTGGC
\end{tabular}

RT-PCR, reverse transcription-polymerase chain reaction; AFP, $\alpha$-fetoprotein; ALB, albumin; CK18, cytokeratin 18; TAT, tyrosine aminotransferase; CYP7A1, cytochrome p450, family 1, member A1.

demonstrated that 5 -azaC significantly increased the hepatic differentiation of embryonic hepatic progenitor HP14.5 cells in the hepatocyte induction medium. The present study assists in the development of effective strategies to induce hepatic progenitor cells differentiation and lays a foundation for the use of progenitor cells as seed cells for liver transplantation in order to treat disease resulting from liver injury.

\section{Materials and methods}

Cell culture and chemicals. HP14.5 cells were isolated from the livers of embryonic mice at day 14.5 post coitus, and immortalized with SV40 large T antigen as described previously (16). HP14.5 cells were cultured in Dulbecco's modified Eagle's medium (DMEM; Gibco Life Technologies, Carlsbad, CA, USA) supplemented with $10 \%$ (v/v) fetal bovine serum (FBS, Gibco Life Technologies), 100 units/ml penicillin, and $100 \mu \mathrm{g} / \mathrm{ml}$ streptomycin at $37^{\circ} \mathrm{C}$ in $5 \% \mathrm{CO}_{2}$. The hepatic differentiation induction medium was composed of $0.1 \mu \mathrm{M}$ Dexmethesone (Dex)/ 10ng/ml Hepatic growth factor (HGF)/20 ng/ml Fibroblast growth factor-4 (FGF4) and $2 \%$ horse serum (HS, Hyclone Laboratories, Inc., Logan UT, USA) in DMEM. Unless indicated otherwise, all chemicals were purchased from Sigma-Aldrich (St. Louis, MO, USA).

Transfection of albumin promoter-driven Gaussia luciferase (ALB-GLuc) reporter and Gaussia luciferase reporter assay. HP14.5 cells were transfected with the pSEB-ALB-GLuc reporter vector (17). To construct pSEB-ALB-GLuc vector, the mouse ALB promoter gene was amplified by polymerase chain reaction (PCR) and subcloned into pSEB-GLuc retroviral vector to drive the expression of Gaussia luciferase. Following $24 \mathrm{~h}$ of transfection, cells were replanted to 24 -well plates and treated with 5-azaC at concentrations of 0, 0.1, 0.2, 0.5, 1, 2, 5, 10 or $20 \mu \mathrm{mol} / 1$, with or without hepatic differentiation induction. Gaussia luciferase possesses a natural secretory signal, which is secreted into the cell medium. Thus, at the indicated time points, the medium was collected in order to detect the activity of Gaussia luciferase, using a Gaussia Luciferase Assay kit (New England Biolabs, Ipswich, MA, USA). Each assay was performed in triplicate and three independent experiments were conducted.
Reverse transcription-quantitative (RT-q)PCR. As previously described (18), the total RNA from each of the HP14.5 cell groups was extracted using the TRIzol (Invitrogen Life Technologies, Carlsbad, CA, USA) method. In order to generate cDNA templates, $10 \mathrm{mcg}$ of total RNA was reverse transcribed with random hexamer pairs using Superscript II reverse transcriptase (Invitrogen Life Technologies, Carlsbad, CA, USA). PCR primers were designed using the Primer3 program to amplify the gene of interest (Table I). qPCR reactions were conducted using a Bio-Rad protocol as follows: $94^{\circ} \mathrm{Cfor}$ 20 seconds, $55^{\circ} \mathrm{C}$ for 20 seconds, and $70^{\circ} \mathrm{C}$ for 20 seconds, for 40 cycles. Plates were read after each cycle. Data were reported as the fold-change with endogenous GAPDH normalization.

Western blot assay. Western blotting was performed as previously described $(18,19)$. Total proteins were extracted from treated HP14.5 cells, which were lysed in radioimmunoprecipitation assay buffer with PMSF (Beyotime Institute of Biotechnology, Shanghai, China). Approximately $20 \mu \mathrm{g}$ of total protein per lane was electrophoretically separated on a 10\% SDS-polyacrylamide gel (Beyotime Institute of Biotechnology) and then transferred to a polyvinylidene fluoride membrane (Millipore, Billerica, MA, USA). The membrane was blocked with $5 \%$ non-fat milk in Tris-buffered saline with Tween-20 (TBST; Beyotime Institute of Biotechnology) at room temperature for $1 \mathrm{~h}$ and incubated with rabbit anti-ALB ployclonal antibody (1:200; cat. no. sc-50536; Santa Cruz Biotechnology, Inc., Dallas, TX, USA) or mouse anti- $\beta$-actin moloclonal antibody (1:200; cat. no sc-47778; Santa Cruz Biotechnology, Inc.) antibodies at $4^{\circ} \mathrm{C}$ overnight. Following washing with TBST, the membrane was probed with the appropriate secondary antibody, conjugated with horseradish peroxidase (Santa Cruz Biotechnology, Inc., Dallas, TX, USA), at room temperature for $1 \mathrm{~h}$. Protein expression was visualized using enhanced Chemiluminescent substrate (Kaiji, Nanjing, China) and exposed under the Syngene GBox Imaging system (Syngene, Cambridge, UK).

Immunofluorescence Staining. Briefly, at 12 days following induction, cells were fixed in ice-cold methanol for $15 \mathrm{~min}$, permeabilized with $1 \%$ NP-40 and blocked with $5 \%$ bovine serum albumin. The cells were then incubated with primary 
A

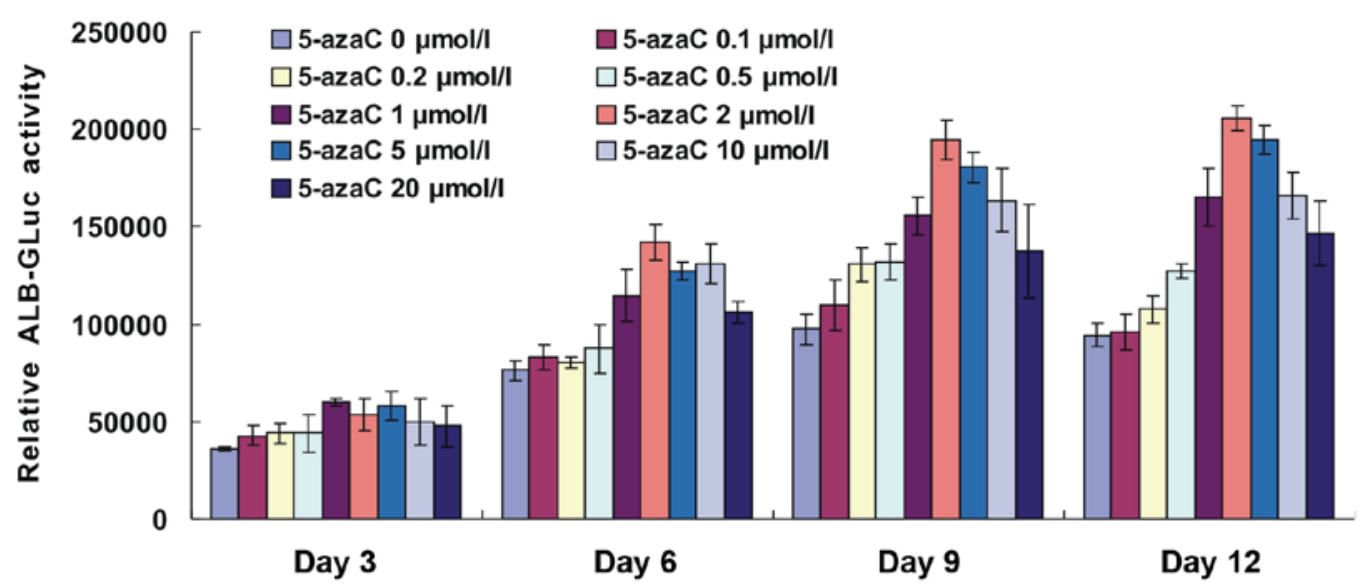

B

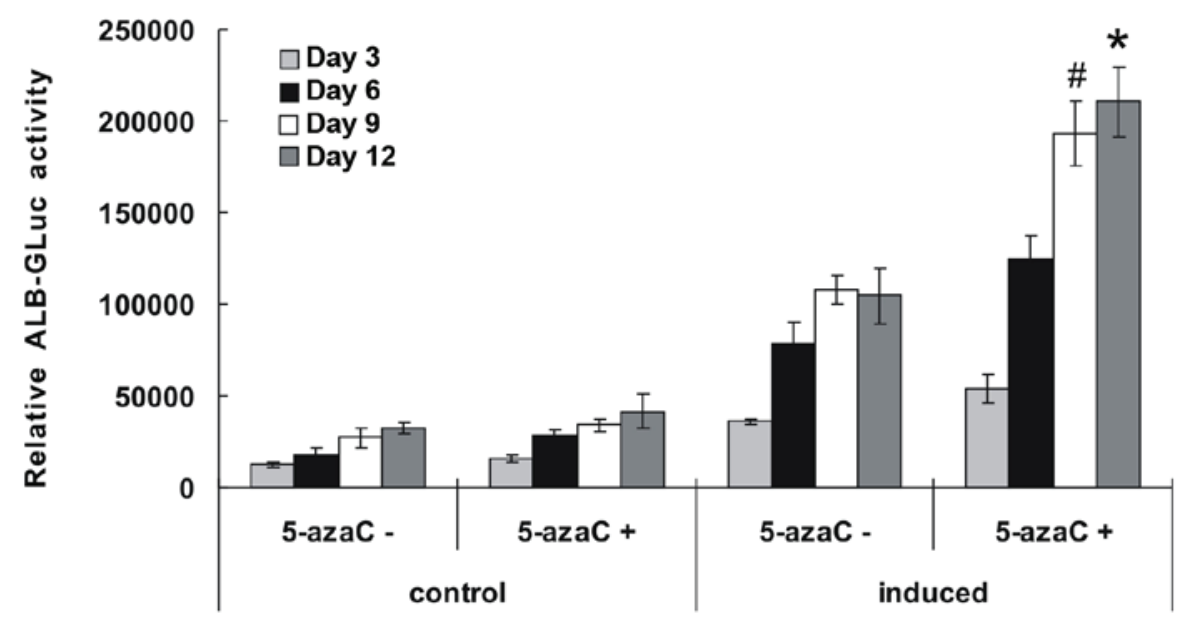

Figure 1.5-azaC increased the ALB-GLuc activity of induced HP14.5 cells. (A) Effect of various concentrations of 5-azaC on ALB-GLuc activity in HP14.5 cells, cultured in a hepatocyte induction medium. Cells were transfected with pSEB-ALB-GLuc plasmid at $24 \mathrm{~h}$ prior to hepatic induction and treated with 5-azaC at concentrations of $0,0.1,0.2,0.5,1,2,5,10$ or $20 \mu \mathrm{mol} / 1$, with hepatic differentiation induction. (B) HP14.5 cells were treated with $2 \mu \mathrm{mol} / 15$-azaC and hepatocyte induction medium. ALB-GLuc activity of HP14.5 cells was detected at 3, 6,9 and 12 days following treatment. "P $<0.05$, compared with the control group and ${ }^{*} \mathrm{P}<0.05$, compared with the group treated with induction medium alone. 5-azaC, 5-azacytidine; ALB-GLuc, albumin promotor-driven Gaussia luciferase.

goat anti-Cytokeratin 18 (CK18; 1:100; cat. no. sc-31700; Santa Cruz Biotechnology, Inc.) or rabbit anti-uridine diphosphate-glucuronosyltransferase 1A (UGT1A) polyclonal antibody (1:100; cat. no. sc-25847; Santa Cruz Biotechnology, Inc.) at $4^{\circ} \mathrm{C}$ overnight, followed by probing with DyLight 594- or 488-labelled secondary antibodies (Jackson ImmunoResearch Laboratories, West Grove, PA, USA) at room temperature for $30 \mathrm{~min}$. Protein expression was examined under a fluorescence microscope (Nikon Intensilight C-HGF1; Nikon, Tokyo, Japan). Samples produced with control IgG were set up as negative controls.

ICG uptake and release. Cells were cultured in 24-well plates. At 12 days following treatment, cells were gently washed with PBS and incubated in $0.5 \mathrm{ml}$ of complete DMEM medium, supplemented with $1 \mathrm{mg} / \mathrm{ml}$ freshly-prepared cardiogreen at $37^{\circ} \mathrm{C}$ for $1 \mathrm{~h}$. DMEM medium was then removed and the samples were gently washed several times with PBS. Green-stained cells were counted as ICG-positive cells under a microscope (Nikon Eclipse Ti-S; Nikon). Cells were then incubated in complete DMEM medium at $37^{\circ} \mathrm{C}$ for $>6 \mathrm{~h}$ in order to assess ICG release, using a microscope $(16,19)$. Ten nonoverlapping images were recorded.
Periodic acid-Schiff (PAS) staining. HP14.5 cells, cultured in 24-well plates, were treated for 12 days. Cells were fixed with $4 \%$ paraformaldehyde for $10 \mathrm{~min}$ and then incubated in $0.5 \%$ periodic acid solution for $5 \mathrm{~min}$. Cells were then rinsed in $\mathrm{ddH}_{2} \mathrm{O}$ for $3 \mathrm{~min}$, incubated with Schiff's reagent for $15 \mathrm{~min}$ and counter-stained with hematoxylin solution for $2 \mathrm{~min}$. Cells were subsequently thoroughly rinsed with tap water. All steps were performed at room temperature $(16,19)$. Ten nonoverlapping visual fields were recorded using a microscope, and cells stained a purple-red color were counted as positive.

Statistical analysis. All data are presented as the mean \pm standard deviation, and were calculated using SPSS 15.0 software (SPSS, Inc., Chicago, IL USA). A two-tailed Student's t-test was used for statistical analysis. $\mathrm{P}<0.05$ was considered to indicate a statistically significant difference.

\section{Results}

ALB-GLuc activity at various 5-azaC concentrations in induced mouse HP14.5 cells. 5-azaC, added at various concentrations to the hepatocyte induction culture medium, induced 
A
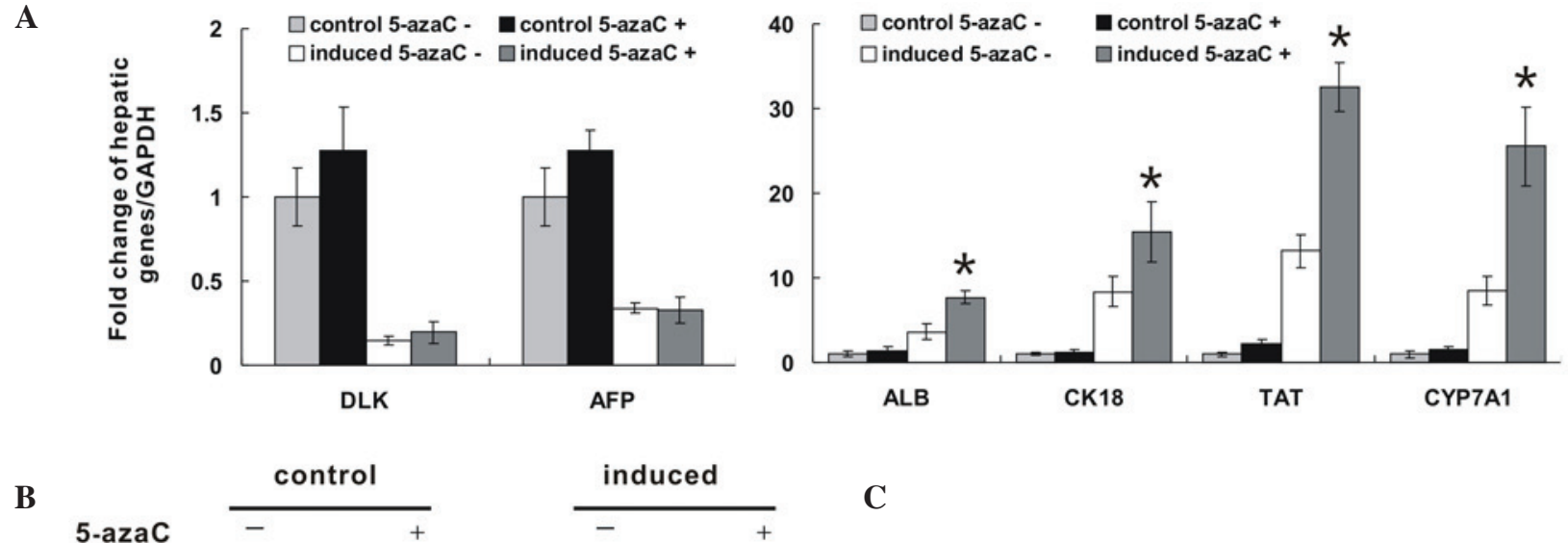

C
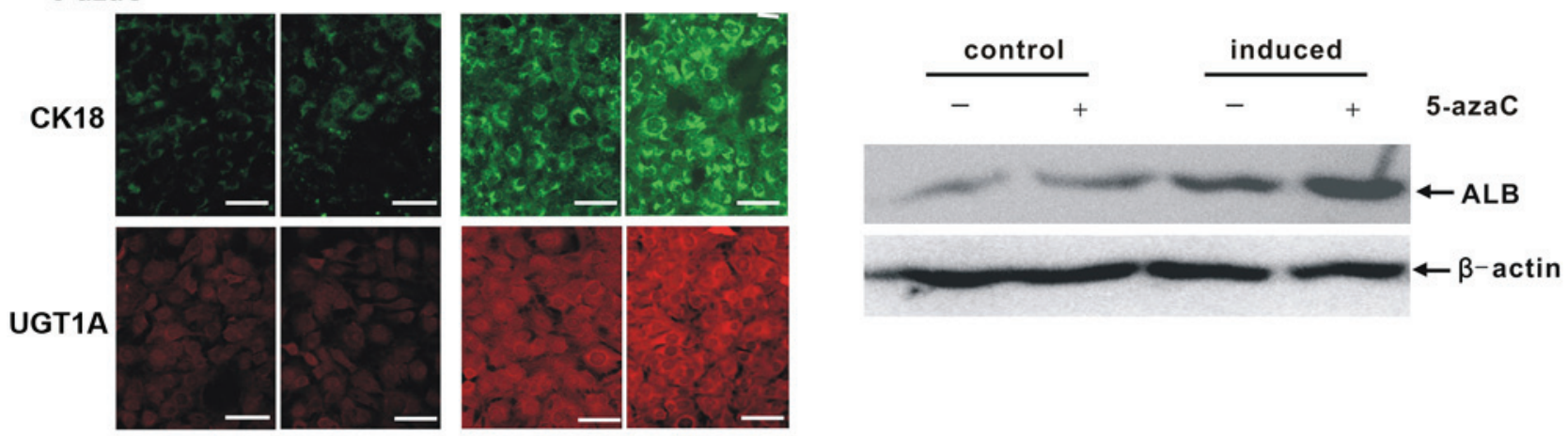

Figure 2. 5-azaC increased the expression of hepatic-associated markers of induced HP14.5 cells. HP14.5 cells were treated with $2 \mu$ mol/1 5 -azaC alone, hepatocyte induction medium alone, or a combination of the two, for 12 days. Untreated cells were used as a control. (A) mRNA expression of hepatic-associated marker genes, DLK, AFP, ALB, CK18, TAT and CYP7A1. Total RNA of cells in the different groups was extracted and reverse-transcribed into cDNA templates. The expression of genes was detected by qPCR and the fold of change was normalized to the expression GAPDH. qPCR results were confirmed in at least three independent experiments ${ }^{*} \mathrm{P}<0.05$, compared with induction medium alone treated group. (B) Protein expression of CK18 and UGT1A was detected using immunofluorescence staining. Scale bar $=200 \mu \mathrm{m}$. (C) Expression of ALB was detected by western blot analysis. Equal loading of the samples was confirmed by $\beta$-actin. 5-azaC, 5 -azacytidine; AFP, $\alpha$-fetoprotein; ALB, albumin; CK18, cytokeratin 18; TAT, tyrosine aminotransferase; CYPA1, cytochrome p450, family 1, member A1; qPCR, quantitative polymerase chain reaction; UGT1A, uridine diphosphate-glucuronyltransferase 1A.

the differentiation of HP14.5 cells. ALB-GLuc is transcribed from the ALB promoter and drives the luciferase reporter gene. Its activity indirectly reflects the level of ALB expression in cells, providing a useful means with which to detect hepatocyte maturation. At 6 days following induction, ALB-GLuc readings were higher in the induced group with 5-azaC than in the induced group without 5-azaC, and exhibited a progressive increase with increasing induction time, reaching a peak following induction for 9-12 days. It was shown that $2 \mu \mathrm{mol} / 15$-azaC was the optimal concentration for hepatic induction (Fig. 1A).

5-azaC enhances ALB-GLuc activity of HP14.5 cells treated with hepatocyte induction culture medium. The ALB-GLuc activity using $2 \mu \mathrm{mol} / 15$-azaC, with or without hepatic induction culture medium was subsequently detected. As shown in Fig. 1B, no significant difference in ALB-GLuc activity was observed between the group treated with 5-azaC alone and the control group. By contrast, 5-azaC significantly increased the ALB-GLuc activity of induced HP14.5 cells.

5-azaC increases the expression of hepatic-associated marker genes of HP14.5 cells in association with induction medium. qPCR results (Fig. 2A) showed that in the group treated with 5 -azaC alone, the expression of various hepatic-associated factors increased slightly, although it was not significantly different compared with that in the control group. Hepatic induction medium induced HP14.5 cells differentiation: The expression of DLK and $\alpha$-fetoprotein (AFP), which are characteristic markers of hepatic stem cells, decreased significantly, while that of ALB, CK18, tyrosine aminotransferase (TAT) and cytochrome P450, family 7, subfamily A, polypeptide 1(CYP7A1), which are mature hepatocyte markers, increased significantly. ALB, CK18, TAT and CYP7A1 expression in the induced group treated with 5 -azaC, was higher than that of the induced group without 5-azaC. Western blotting results were consistent with these findings. 5-azaC treatment alone did not affect the expression of ALB protein, while it enhanced the expression of this protein in an induced environment (Fig. 2B). Immunofluorescence images demonstrated expression of the mature hepatocyte markers, CK18 and UGT1A, in cytoplasm, and no difference was detected between the group treated with 5-azaC alone and the control group. By contrast, the expression of these markers in the 5-azaC induced group was significantly higher than that in the induced group without 5-azaC ( $\mathrm{P}=0.008597$; Fig. 2C). Thus, the results suggested that 5 -azaC alone is insufficient to induce hepatic progenitor cell differentiation. However, it does stimulate hepatic maturation and differentiation within the appropriate induced environment.

5-azaC enhances the mature hepatic function of HP14.5 cells in association with induction. Mature hepatic cells are known to metabolize ICG, and ICG uptake may therefore be used for 
A
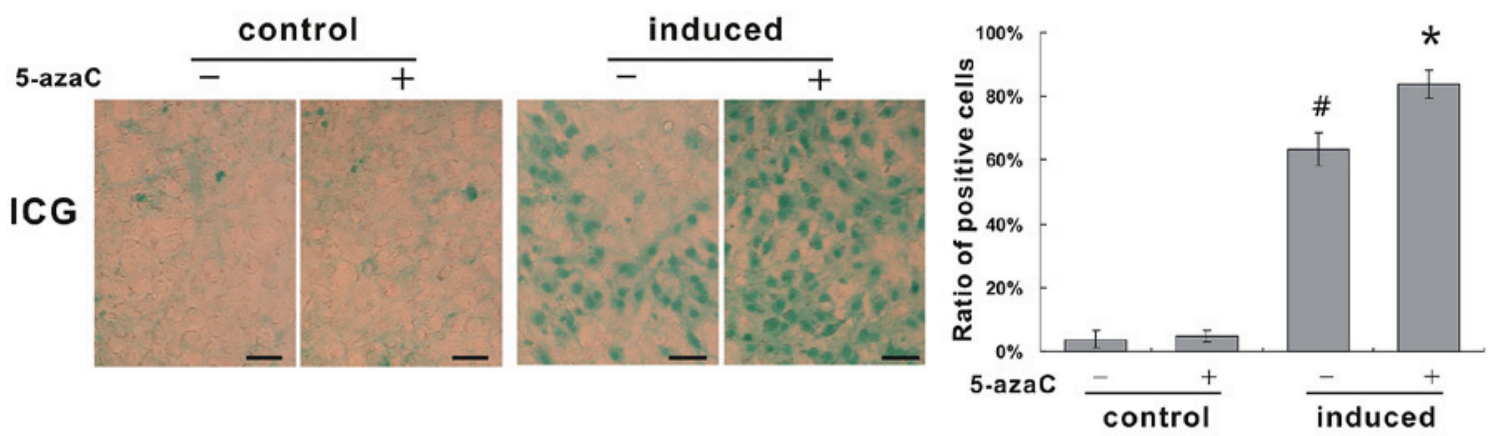

B
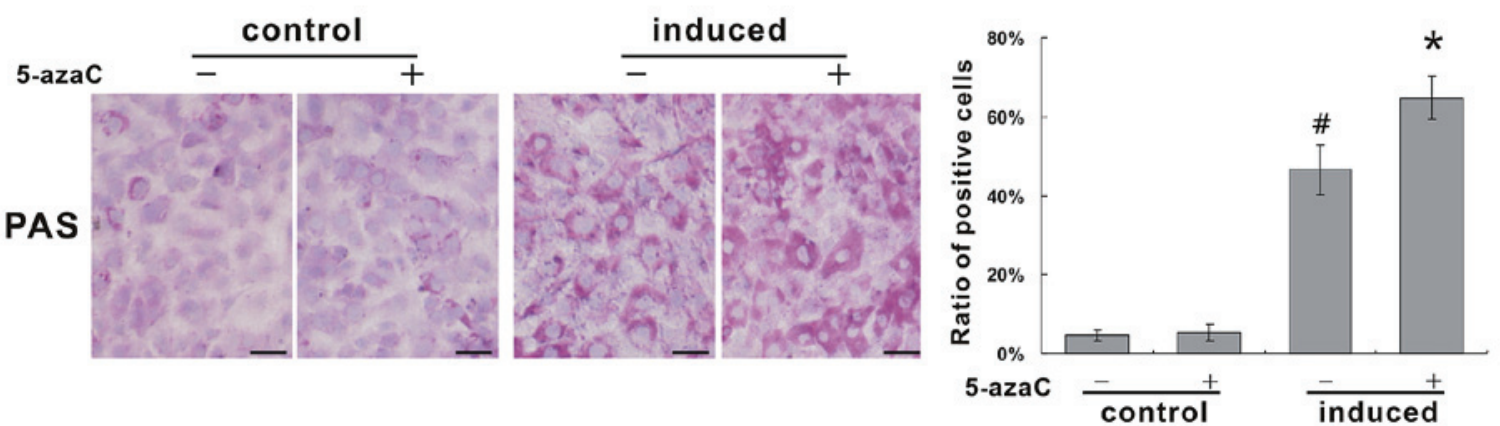

Figure 3. 5-azaC enhanced the mature hepatic function of HP14.5 cells in association with hepatic induction. Cells were treated as described in Figure 2. (A) Transport and metabolic function was evaluated by an ICG uptake assay. Positive cells exhibited a green-stained nucleus. (B) Glycogen storage and accumulation function in the induced HP14.5 cells was measured using a PAS staining method. Purple staining in the cell plasma indicated glycogen accumulation. Three independent experiments were performed. Scale bar $=200 \mu \mathrm{m}$. Six nonoverlapping visual fields were randomly selected and cells were counted to calculate the ratio of positive to negative cells. ${ }^{*} \mathrm{P}<0.05$, compared with the control group and ${ }^{*} \mathrm{P}<0.05$, compared with the group treated with induction medium alone. 5-azaC, 5-Azacytadine; ICG, indocyanine green; PAS, periodic acid-Schiff.

the identification of differentiated hepatocytes in vitro $(20,21)$. The ratios of ICG-positive cells to ICG-negative cells in the non-induced control group and in the group treated with 5 -azaC alone, were $3.9 \pm 2.8 \%$ and $4.8 \pm 1.9 \%$, respectively, and no significant difference was detected between the two groups. Following 12 days of induction, the ratio of ICG-positive to ICG-negative cells was $63.4 \pm 5.1 \%$, which was significantly higher than that of the control group, while it was significantly

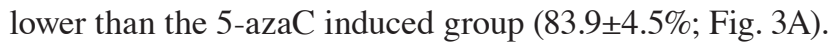

Glycogen synthesis function is an important indicator in the evaluation of hepatic differentiation. Synthetic glycogen appears a purple-red color in the cytoplasm, upon PAS staining (22). The ability of HP14.5 cells to synthesize glycogen was weak, with a ratio of PAS-positive to PAS-negative cells of $4.6 \pm 1.4 \%$. Treatment with 5 -azaC alone, did not significantly increase this ratio $(5.3 \pm 2.1 \%)$. At 12 days following induction, the ratio of PAS-positive to PAS-negative HP14.5 cells increased to $46.6 \pm 6.3 \%$, while the ratio in the induced group treated with 5 -azaC was $64.7 \pm 5.4 \%$, which was significantly higher than that in the induced group without 5-azaC. There was widespread purple-red throughout the cytoplasm, which was of a deeper color than that in the induced group (Fig. 3B). These results demonstrated that 5 -azaC enhances the metabolic and synthetic function of HP14.5 cells in association with hepatocyte induction.

\section{Discussion}

Hepatic progenitor cells are a form of stem cell, which are able to self-proliferate; differentiate into hepatocytes and biliary epithelial cells, and participate in liver repair and reconstruction. They may also be an important cell source for hepatic cell transplantation $(7,23,24)$. Current research on hepatic stem cells remains at the theoretical and experimental stage. Extrahepatic sources of hepatic stem cells, including embryonic stem cells, hematopoietic stem cells and bone marrow MSCs are able to differentiate into hepatocyte-like cells that express hepatocyte-specific genes or exhibit partial hepatic cell function of regeneration in vivo $(25,26)$. However, the differentiation efficiency and substitution function of these cells in vivo is far less than hepatic progenitor cells from embryos $(27,28)$. The process of differentiation of stem cells into hepatocytes, includes bipotential hepatic progenitor cells. Embryonic hepatic progenitor cells are precursor cells, and may differentiate into hepatocytes or biliary epithelial cells. The intermediate bipotential state is therefore required during the differentiation of other types of stem cells into mature hepatocytes $(26,29,30)$.

Hepatocyte differentiation is periodically regulated by different signals. In particular, the participation of associated cytokines is required to facilitate stem cell differentiation into hepatic endoderm (31-33). At the hepatic progenitor cell stage, in which cells exhibit bi-directional differentiation potential, the gene regulation system sends different instructions to the cells, in order to induce their differentiation into hepatocytes and biliary epithelial cells, thus producing a unique hepatic tissue morphology and function. It has been demonstrated that upregulating the expression of differential-related genes may improve the therapeutic efficacy of hepatic stem cells transplantation $(34,35)$. Therefore, stable and efficient hepatocyte 
resources obtained from hepatic progenitor cells may significantly improve the efficiency and biosafety profile of liver cell transplantation.

5 -azaC is a type of cytosine chemical analogue, which is able to interfere with the physiological function of DNA by embedding in this molecule in order to exert cytotoxic and antitumor effects $(10,36)$. 5-azaC has been used for the treatment of breast cancer, colon cancer, melanoma and acute myelogenous leukemia in clinical practice (37-39). 5-azaC also functions as a DNA methylation inhibitor. It combines with the methylation enzyme and inactivates it, which leads to hypomethylated DNA, thereby enhancing gene expression. In 1995, Wakitani et al (40) reported that 5-azaC induces the differentiation of bone marrow MSC into cardiomyocytes. 5 -azaC has been widely used in a variety of directional stem cell induction. MSCs treated with 5-azaC in vitro express cardiac-specific structural protein (atrial natriuretic peptide, brain natriuretic peptide and $\alpha-/ \beta$-myosin heavy chain) and myocardiocyte-specific transcription factors (GATA4 and Nkx2.5/Csx), and partly improve myocardial systolic ventricular pressure and tension following transplantation into freezing damaged rat myocardium in vivo (41-43). Furthermore, brief exposure to 5 -azaC induces pig dermal fibroblast reprogramming into insulin secreting cells (44). A high concentration $(10 \mu \mathrm{mol} / \mathrm{l})$ of 5 -azaC has been reported to enhance the induction of adipose-derived stem cell differentiation into myogenic cardiogenic cells (45).

5-azaC mediated-inhibition of DNA methylation is widespread rather than cell-specific. However, there are a number of studies that have investigated the effect of 5-azaC on hepatocyte differentiation and hepatic tumors. It has been reported that 5-azaC inhibits HepG2 and Hep3B liver tumor cell proliferation, induces apoptosis, and promotes their maturation and differentiation (46). The present study showed that 5-azaC exerts certain inducing differentiation effects, when administered at a suitable concentration. However, when treated with a high concentration condition of 5-azaC, the ALB-GLuc activity of induced HP14.5 cells decreased. This phenomenon may be due to higher doses of 5-azaC directly inhibiting cell proliferation, and mediating cell cytotoxicity by embedding into DNA and RNA $(47,48)$. By contrast, lower doses of 5-azaC primarily inhibit DNA methylation, resulting in the recovery of gene normal expression $(10,49,50)$. Concomitantly, it was found that hepatic-associated markers of HP14.5 cells treated with 5-azaC alone, did not increase significantly, suggesting that DNA hypomethylation is not the only factor that determines gene expression during the hepatic cell differentiation process; the appropriate induced culture conditions and the microenvironment also have an effect on transcriptional control. Similarly, MSCs may be induced to differentiate into cardiomyocytes by 5 -azaC, although it is difficult to to obtain functional beating myocardial cells (51). DLK and AFP proteins are markers of hepatic stem cells $(52,53)$. If 5-azaC promotes the maturational differentiation of induced hepatic cells, the expression of these stem cell markers should decrease. However, as 5-azaC could inhibit DNA methylation, stem cell marker gene expression in the 5-azaC induced group remained at the same level as that in the induced group without 5-azaC treatment. The expression of CK18 and ALB, which are markers of mature hepatocytes, was higher in the induced group with 5-azaC than in the induced group without 5-azaC. It was hypothesized that the different conformations of DNA cpG islands may determine the extent of the effect of 5-azaC, which functions primarily via modification of methylation $(54,55)$. This proposal requires further investigation.

In conclusion, The present study demonstrated that 5-azaC synergistically promotes the hepatic differentiation of HP14.5 cells, significantly increases the expression of hepatic-associated marker genes, and enhances the ICG metabolism and glycogen synthesis function of these cells. The current study provides a basis for the clinical application of hepatic progenitor cells in liver disease. 5-azaC is known to induce differentiation into myocardiocytes. The present study demonstrates that it is also involved in the terminal maturation and differentiation of induced hepatocytes, suggesting a wider role for this molecule as an inducing agent.

\section{Acknowledgements}

This study was supported by grants from the National Natural Science Foundation of China (grant no. 81100309).

\section{References}

1. Samuel D, Colombo M, El-Serag H, Sobesky R and Heaton N: Toward optimizing the indications for orthotopic liver transplantation in hepatocellular carcinoma. Liver Transpl 17 (Suppl 2): S6-S13, 2011.

2. Adam R and Hoti E: Liver transplantation: The current situation. Semin Liver Dis 29: 3-18, 2009.

3. Oertel M. Fetal liver cell transplantation as a potential alternative to whole liver transplantation? J Gastroenterol 46: 953-965, 2011.

4. Hughes RD, Mitry RR and Dhawan A: Current status of hepatocyte transplantation. Transplantation 93: 342-347, 2012.

5. Haridass D, Narain N and Ott M: Hepatocyte transplantation: Waiting for stem cells. Curr Opin Organ Transplant 13: 627-632, 2008.

6. Sancho-Bru P, Najimi M, Caruso M, et al: Stem and progenitor cells for liver repopulation: Can we standardise the process from bench to bedside? Gut 58: 594-603, 2009.

7. Kakinuma S, Nakauchi $\mathrm{H}$ and Watanabe $\mathrm{M}$ : Hepatic stem/progenitor cells and stem-cell transplantation for the treatment of liver disease. J Gastroenterol 44: 167-172, 2009.

8. Sharma AD, Cantz T, Vogel A, et al: Murine embryonic stem cell-derived hepatic progenitor cells engraft in recipient livers with limited capacity of liver tissue formation. Cell Transplant 17: 313-323, 2008.

9. Tomiyama K, Miyazaki M, Nukui M, et al: Limited contribution of cells of intact extrahepatic tissue origin to hepatocyte regeneration in transplanted rat liver. Transplantation 83: 624-630, 2007.

10. Venturelli S, Berger A, Weiland T, et al: Differential induction of apoptosis and senescence by the DNA methyltransferase inhibitors 5-azacytidine and 5-aza-2'-deoxycytidine in solid tumor cells. Mol Cancer Ther 12: 2226-2236, 2013.

11. Kang PL, Chen CH, Chen SY, et al: Nano-sized collagen I molecules enhanced the differentiation of rat mesenchymal stem cells into cardiomyocytes. J Biomed Mater Res A 101: 2808-2816, 2013.

12. Moscoso I, Centeno A, López E, et al: Differentiation 'in vitro' of primary and immortalized porcine mesenchymal stem cells into cardiomyocytes for cell transplantation. Transplant Proc 37: 481-482, 2005.

13. Rosca AM and Burlacu A: Effect of 5-azacytidine: Evidence for alteration of the multipotent ability of mesenchymal stem cells. Stem Cells Dev 20: 1213-1221, 2011.

14. De Carvalho DD, You JS and Jones PA: DNA methylation and cellular reprogramming. Trends Cell Biol 20: 609-617, 2010.

15. Kamiya A, Gonzalez FJ and Nakauchi H: Identification and differentiation of hepatic stem cells during liver development. Front Biosci 11:1302-1310, 2006.

16. Bi Y, He Y, Huang JY, et al: Induced maturation of hepatic progenitor cells in vitro. Braz J Med Biol Res 46: 559-566, 2013. 
17. Bi Y, Huang J, He Y, et al: Wnt antagonist SFRP3 inhibit the differentiation of mouse hepatic progenitor cells. J Cell Biochem 108: 295-303, 2009.

18. Bi Y, Gong M,He Y, Wei X, Chen J and Li T: Adenovirus-mediated RAR- $\beta$ over-expression enhances ATRA-induced neuronal differentiation of rat mesenchymal stem cells. Arch Med Sci 9 314-322, 2013.

19. He Y, Zhou JW, Xu L, Gong MJ, He TC and Bi Y: Comparison of proliferation and differentiation potential between mouse primary hepatocytes and embryonic hepatic progenitor cells in vitro. Int J Mol Med 32: 476-484, 2013.

20. Yamada T, Yoshikawa M, Kanda S, et al: In vitro differentiation of embryonic stem cells into hepatocyte-like cells identified by cellular uptake of indocyanine green. Stem Cells 20: 146-154, 2002.

21. Yoshie S, Ito J, Shirasawa S, et al: Establishment of novel detection system for embryonic stem cell-derived hepatocyte-like cells based onnongenetic manipulation with indocyanine green. Tissue Eng Part C Methods 18: 12-20, 2012.

22. König M, Bulik S and Holzhütter HG: Quantifying the contribution of the liver to glucose homeostasis: A detailed kinetic model of human hepatic glucose metabolism. PLoS Comput Biol 8: e1002577, 2012.

23. Weiss TS, Lichtenauer M, Kirchner S, et al: Hepatic progenitor cells from adult human livers for cell transplantation. Gut 57: 1129-1138, 2008.

24. Sangan CB and Tosh D: Hepatic progenitor cells. Cell Tissue Res 342: 131-137, 2010.

25. Sokal EM: From hepatocytes to stem and progenitor cells for liver regenerative medicine: Advances and clinical perspectives. Cell Prolif 44 (Suppl 1): 39-43, 2011.

26. Heo J, Factor VM, Uren T, et al: Hepatic precursors derived from murine embryonic stem cells contribute to regeneration of injured liver. Hepatology 44: 1478-1486, 2006.

27. Zhang W, Li W, Liu B, Wang P, Li W and Zhang H: Efficient generation of functional hepatocyte-like cells from human fetal hepatic progenitor cells in vitro. J Cell Physiol 227: 2051-2058, 2012.

28. You N, Liu W, Zhong X, Dou K and Tao K: Possibility of the enhanced progression of fetal liver stem/progenitor cells therapy for treating end-stage liver diseases by regulating the notch signaling pathway. Arch Med Res 43: 585-587, 2012.

29. Chen Q, Khoury M, Limmon G, Choolani M, Chan JK and Chen J: Human fetal hepatic progenitor cells are distinct from, but closely related to, hematopoietic stem/progenitor cells. Stem Cells 31:1160-1169, 2013.

30. Friedman JR and Kaestner KH: On the origin of the liver. J Clin Invest 121: 4630-4633, 2011.

31. Du Z, Wei C, Yan J, et al: Mesenchymal stem cells overexpressing $\mathrm{C}$-X-C chemokine receptor type 4 improve early liver regeneration of small-for-size liver grafts. Liver Transpl 19: 215-225, 2013.

32. Haga S, Ozaki M, Inoue $\mathrm{H}$, et al: The survival pathways phosphatidylinositol-3 kinase (PI3-K)/phosphoinositide-dependent protein kinase 1 (PDK1)/Akt modulate liver regeneration through hepatocyte size rather than proliferation. Hepatology 49: 204-214, 2009.

33. Kwon HJ, Won YS, Yoon YD, et al: Vitamin D3 up-regulated protein 1 deficiency accelerates liver regeneration after partial hepatectomy in mice. J Hepatol 54: 1168-1176, 2011.

34. Turner RA, Wauthier E, Lozoya O, et al: Successful transplantation of human hepatic stem cells with restricted localization to liver using hyaluronan grafts. Hepatology 57: 775-784, 2013.

35. Lozoya OA, Wauthier E, Turner RA, et al: Regulation of hepatic stem/progenitor phenotype by microenvironment stiffness in hydrogel models of the human liver stem cell niche. Biomaterials 32: 7389-7402, 2011.

36. Ding YB, Long CL, Liu XQ, et al: 5-aza-2'-deoxycytidine leads to reduced embryo implantation and reduced expression of DNA methyltransferasesand essential endometrial genes. PLoS One 7: e45364, 2012.
37. Ghoshal K and Bai S: DNA methyltransferases as targets for cancer therapy. Drugs Today (Barc) 43: 395-422, 2007.

38. Buckstein R, Yee K, Wells RA; Canadian Consortium on Evidence-based Care in MDS: 5-Azacytidine in myelodysplastic syndromes: A clinical practice guideline. Cancer Treat Rev 37: 160-167, 2011.

39. Deng G, Kakar S, Okudiara K, Choi E, Sleisenger MH and Kim YS: Unique methylation pattern of oncostatin $\mathrm{m}$ receptor gene in cancers of colorectum and other digestive organs. Clin Cancer Res 15: 1519-1526, 2009.

40. Wakitani S, Saito T and Caplan AI: Myogenic cells derived from rat bone marrow mesenchymal stem cells exposed to 5-azacytidine. Muscle Nerve 18: 1417-1426, 1995.

41. Makino S, Fukuda K, Miyoshi S, et al: Cardiomyocytes can be generated from marrow stromal cells in vitro. J Clin Invest 103: 697-705, 1999.

42. Yeh HY, Liu BH and Hsu SH: The calcium-dependent regulation of spheroid formation and cardiomyogenic differentiation for MSCs on chitosan membranes. Biomaterials 33: 8943-8954, 2012.

43. Naeem N, Haneef K, Kabir N, Iqbal H, Jamall S and Salim A: DNA methylation inhibitors, 5-azacytidine and zebularine potentiate the transdifferentiation of rat bone marrow mesenchymal stem cells into cardiomyocytes. Cardiovasc Ther 31: 201-209, 2013.

44. Pennarossa G, Maffei S, Campagnol M, Rahman MM, Brevini TA and Gandolfi F: Reprogramming of pig dermal fibroblast into insulin secreting cells by a brief exposure to 5-aza-cytidine. Stem Cell Rev 10: 31-43, 2014

45. Ravichandran R, Venugopal JR, Mueller M, et al: Buckled structures and 5-azacytidine enhance cardiogenic differentiation of adipose-derived stem cells. Nanomedicine (Lond) 8: 1985-1997, 2013

46. Wang XM, Wang X, Li J and Evers BM: Effects of 5-azacytidine and butyrate on differentiation and apoptosis of hepatic cancer cell lines. Ann Surg 227: 922-931, 1998.

47. Guo Y, Engelhardt M, Wider D, Abdelkarim M and Lübbert M: Effects of 5-aza-2'-deoxycytidine on proliferation, differentiation and $\mathrm{p} 15 / \mathrm{INK} 4 \mathrm{~b}$ regulation of human hematopoietic progenitor cells. Leukemia 20: 115-121, 2006.

48. Zhou Y and Lu Q: DNA methylation in T cells from idiopathic lupus and drug-induced lupus patients. Autoimmun Rev 7: 376-383, 2008.

49. Dhawan D, Ramos-Vara JA, Hahn NM, et al: DNMT1: An emerging target in the treatment of invasive urinary bladder cancer. Urol Oncol 31: 1761-1769, 2013.

50. Stern-Straeter J, Bonaterra GA, Juritz S, et al. Evaluation of the effects of different culture media on the myogenic differentiation potential of adipose tissue- or bone marrow-derived human mesenchymal stem cells. Int J Mol Med 33: 160-170, 2014.

51. Ramesh B, Bishi DK, Rallapalli S, Arumugam S, Cherian KM and Guhathakurta S: Ischemic cardiac tissue conditioned media induced differentiation of human mesenchymal stem cells into early stage cardiomyocytes. Cytotechnology 64: 563-575, 2012.

52. Nishina H: hDlk-1: A cell surface marker common to normal hepatic stem/progenitor cells and carcinomas. J Biochem 152: $121-123,2012$.

53. Kuhlmann WD and Peschke P: Hepatic progenitor cells, stem cells, and AFP expression in models of liver injury. Int J Exp Pathol 87: 343-359, 2006.

54. Yang J, Corsello TR and Ma Y: Stem cell gene SALL4 suppresses transcription through recruitment of DNA methyltransferases. J Biol Chem 287: 1996-2005, 2012.

55. Mi XB and Zeng FQ: Hypomethylation of interleukin- 4 and -6 promoters in T cells from systemic lupus erythematosus patients. Acta Pharmacol Sin 29: 105-112, 2008. 